\title{
Consumo televisivo e interacción en redes sociales entre jóvenes seguidores de la serie Élite
}

\section{Television viewing and interaction on social networking sites among Elite series' young followers}

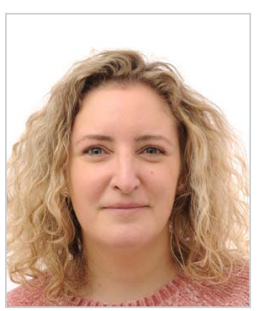

Aurora Forteza Martínez. Doctoranda en el Programa de Doctorado Interuniversitario en Comunicación conjunto de las universidades de Sevilla, Málaga, Huelva y Cádiz en la rama de Educomunicación y Alfabetización Mediática. Graduada en Magisterio en Educación Primaria con mención en Lenguas Extranjeras por la Universidad Camilo José Cela y Diplomada en Magisterio en Educación Infantil por la Universidad de Castilla-La Mancha. Máster en Tecnologías de la Información y la Comunicación para la Educación y el Aprendizaje Digital por la Universidad Antonio de Nebrija. Universidad de Huelva, España

aurora.forteza@alu.uhu.es

ORCID: 0000-0001-9680-5927

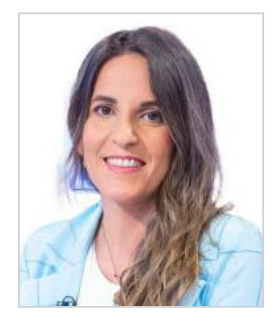

Patricia de Casas Moreno. Doctora por el Programa de Doctorado Interuniversitario en Comunicación conjunto de las universidades de Sevilla, Málaga, Huelva y Cádiz en la rama de Educomunicación. Licenciada en Comunicación por la rama de Periodismo en el Centro Andaluz de Estudios Empresariales (CEADE), especializada en comunicación 2.0 y graduada en el Máster de Comunicación y Educación Audiovisual por la Universidad de Huelva.

Universidad de Huelva, España

pcasas@nebrija.es

ORCID: 0000-0003-1205-8106

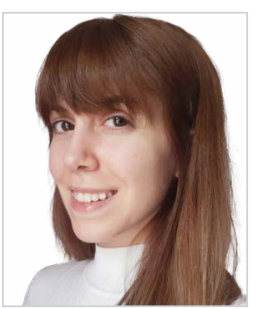

Arantxa Vizcaíno Verdú. Investigadora Predoctoral (FPU) y Doctoranda en el programa Interuniversitario de Comunicación en la línea de Educomunicación y Alfabetización Mediática (UHU). Magíster en Comunicación y Educación Audiovisual por la Universidad de Huelva (2017) y Premio Extraordinario de Trabajo Fin de Máster (2016-2017). Graduada en Publicidad y Relaciones Públicas por la Universidad de Alicante (2016). Editora Asociada de la revista 'Comunicar.' Miembro del Grupo Ágora (HUM-648), el Grupo Comunicar y RRPP de la Red Alfamed.

Universidad de Huelva, España

arantxa.vizcaino@dedu.uhu.es

ORCID: 0000-0001-9399-2077

\section{Cómo citar este artículo:}

Forteza Martínez, A.; De Casas Moreno, P. y Vizcaíno Verdú, A. (2021). Consumo televisivo e interacción en redes sociales entre jóvenes seguidores de la serie Élite. Doxa Comunicación, 33, pp. 217-234. 
Recibido: 01/05/2021 - Aceptado: 07/09/2021 - En edición: 20/09/2021 - Publicado: 01/12/2021

\section{Resumen:}

El ecosistema audiovisual actual está evolucionando a un ritmo vertiginoso. Las nuevas formas de interacción narrativa a través de las redes sociales (RRSS) han generado una revolución en los medios de comunicación en general y en la televisión en particular, concediendo la posibilidad de acceder a los contenidos a través de diferentes formatos y canales. En este sentido, el presente trabajo analiza los hábitos de consumo de la población adolescente ante los nuevos formatos narrativos y su función como prosumidores frente a las RRSS. Para ello, se ha seleccionado la serie de ficción "Élite" como parte de la muestra de estudio. De este modo, se ha llevado a cabo una metodología mixta por medio del análisis de contenido de la serie en busca de las temáticas emergentes, y de encuestas con la colaboración de una muestra de 505 jóvenes españoles de entre 12-18 años. Los resultados apuntan hacia una preferencia por ver series de televisión, siendo las plataformas de pago el medio más frecuente de uso; además, los jóvenes muestran un interés en temáticas relacionadas con los adolescentes. Por último, los encuestados registran una participación constante a través de las diferentes cuentas de RRSS, afirmando indagar sobre la serie de ficción.

\section{Palabras clave:}

Adolescentes; televisión; series; hábitos televisivos; Redes Sociales.
Received: 01/05/2021- Accepted: 07/09/2021- Early access: 20/09/2021 - Published: 01/12/2021

\section{Abstract:}

Today's audiovisual ecosystem is evolving at a dizzying pace. The new forms of interactive storytelling through social networking sites (SNS) have generated a revolution within the communication media in general, and particularly within television, making it possible to access content through different formats and channels. In this respect, the present study analyses the viewing habits of the adolescents faced with the new storytelling formats and their role as prospective consumers with regard to the SNS. To understand this process, we selected the fictional series called "Elite" as the sample. We performed a mixed methodology through content analysis of the series in search of emerging themes, and we also conducted a survey where participated 505 Spanish young people between the ages of 12-18 years. The results showed a preference for viewing television series, where payed television platforms were the most frequently used media; the young people also expressed an interest in themes relating to adolescents. Finally, respondents evidenced a continuous involvement through different SNS profiles, expressing that they used to look for information on fiction series.

\section{Keywords:}

Teenagers; television; series; TV viewing habits; social networking sites.

\section{Introducción}

En la actualidad, la influencia que de las Redes Sociales (RRSS) resulta evidente, en tanto que facilitan a las personas interactuar con otros usuarios (Oliva-Marañón, 2012). Esta situación genera nuevas formas de socialización a través de Internet, distinguida por su inmediatez y efectividad (Díaz-Gandasegui, 2011). Si bien, las RRSS no solo están concebidas para establecer relaciones personales, sino para abordar propósitos dentro del ámbito laboral, académico o político (Flores-Cueto et al., 2009).

Uno de los ámbitos de mayor repercusión de estas plataformas es el entorno audiovisual. Los telespectadores, hoy en día, tienen la posibilidad de decidir no solo qué quieren ver, sino cuándo y cómo disfrutar de su programación preferida, expresando y compartiendo opiniones (Ruano-López et al., 2016). Además, dentro de la sociedad postdigital, se presta atención a la actitud que presentan los jóvenes frente al uso que estos hacen de las RRSS (Gil-Quintana \& Fernández-Galiano-Amorós, 2020).

Asimismo, es fundamental estudiar cuáles son los estereotipos que de los adolescentes se genera a través de las series de televisión, los roles que estos tratan de imitar, así cómo el modo en que interactúan con esos modelos (Sánchez-Noriega, 1997). A través de estos medios se forma una imagen de los jóvenes y estos adoptan estas representaciones e interactúan con ellas (Masanet \& Fedele, 2019). En consonancia a las series dirigidas al público adolescente, estas suelen estar entre la programación más seguidas escogida, debido a que poseen una doble función social y socializadora: por un lado, generan vínculos con el grupo de 
iguales al compartir los contenidos de estas series y, por otro lado, el reconocimiento con los personajes que se muestran en las series a través de las diferentes historias planteadas (Fedele, 2011).

Por ello, en este estudio se pretende saber cuáles son las costumbres que los jóvenes tienen ante los medios, especialmente frente a los formatos narrativos actuales, así como estudiar su figura como prosumidores en las RRSS.

\section{Nuevas formas audiovisuales: el desarrollo de la televisión}

La televisión ha experimentado un gran cambio con respecto a cómo se conocía hace una década debido a su fusión con Internet. En los últimos años, la forma de ver televisión se ha modificado enormemente. Este hecho ha ocurrido, en gran medida, por los progresos tecnológicos, por el nuevo rol que los espectadores han asumido y por la irrupción de los contenidos audiovisuales digitales. Además, cabe apuntar a la irrupción de plataformas digitales online y a la carta, las cuales han supuesto una nueva realidad en contraposición a los medios tradicionales (Capapé, 2020). El apogeo de los contenidos en Internet ha favorecido la producción de apartados específicos en los cuales se integra programación específica, fomentando que estos sean distribuidos a través de las RRSS (Jódar-Marín, 2019). Por ello, la realidad audiovisual se encuentra inmersa en una etapa de cambios como consecuencia del proceso de digitalización, además del cambio de comportamiento de la audiencia, sobre todo entre los adolescentes (Crusafon et al., 2020).

Esto ha provocado el alza de una televisión online e interactiva, facilitando un uso más individual ante la diversidad de pantallas (Diego-González et al., 2014). Actualmente, el acceso a la televisión se puede efectuar a través de múltiples dispositivos, suscitando nuevas manifestaciones en materia de hábitos de consumo y potenciando la interactividad y la personalización (Gallego, 2013). Esta forma de ver la televisión se ha transformado en una práctica de interacción con las RRSS, fomentando el uso de una segunda pantalla. Esto hace referencia al término de competencia multitarea o multitasking, concepto acuñado por Henry Jenkins (2006), definido como una competencia adicional, que posibilita la administración de diferentes habilidades y que guardan una estrecha relación con la memoria temporal del cerebro humano. Asimismo, se alza como una capacidad que tienen los jóvenes para prestar atención a diferentes acciones o elementos a la vez debido al entorno mediático en el que se desenvuelven (Zorrilla-Abascal, 2012). A ello se le suma un proceso de convivencia entre lo online y lo offline (Tse, 2016).

Numerosas investigaciones han señalado que la televisión tradicional ha sido relegada a un segundo plano por parte de los adolescentes (Burgess \& Green, 2009; Galán \& del-Pino, 2010; Morimono \& Friedland, 2011, Prado, 2013, Pavón-Arrizabalaga et al., 2016). Sin embargo, continúan siendo los principales usuarios activos de las producciones audiovisuales. La industria televisiva se ha visto obligada a realizar transformaciones en la creación y distribución de estos productos (Lamelo-Varela, 2016).

Las comunidades virtuales, las RRSS y la actitud de los consumidores implican nuevas formas de ver los contenidos televisivos (Anstead \& O’Loughlin, 2011; Norman, 2012). Esta nueva representación es conocida como la televisión social, definida como "the social practice of commenting on television shows with peers, friends and unknown people, who are all connected together, through various digital devices" (Selva, 2016, p.160). Asimismo, esta se identifica porque se define por la información proporcionada a los espectadores que se mantienen actualizados gracias a la información y se mantienen actualizados gracias a plataformas como Facebook y Twitter, o mediante aplicaciones de comunicación instantánea, como es el caso de WhatsApp (Pereira-López et al., 2019). 
En suma, la televisión general vive inmersa en un periodo de transformación, lo que conlleva una profunda atención en aspectos relacionados con el control sobre el uso multipantalla, aceptar la dispersión de los telespectadores o fomentar la armonía entre la televisión tradicional con las nuevas formas de consumo en streaming (Saavedra-Llamas et al., 2020). El proceso de digitalización fomenta diferentes alternativas a la hora de ofrecer nuevos contenidos en distintos formatos con el fin de cubrir las necesidades de los espectadores (Medina et al., 2019).

\section{Plataformas de interacción de contenidos: RRSS}

La revolución digital ha supuesto un enorme choque con los modelos tradicionales de comunicación y las relaciones sociales, provocada por la facilidad de acceso y el uso de Internet entre los adolescentes (Rial et al., 2014). En este contexto, tal y como afirman Hernández et al., las RRSS "se han convertido en un fenómeno irreversible, en constante despliegue de posibilidades para las relaciones con otras personas y aluden a que en una red social pueden encontrar con quienes comparten intereses, preocupaciones o necesidades" (2017, p. 21).

Según diferentes estudios, Facebook, Twitter o YouTube ofrecen un gran número de recursos para los individuos, donde destaca la interacción con su entorno más inmediato, hecho que les permite compartir aficiones o gustos con los usuarios de estas RRSS (Brenley \& Coven, 2018; Awidi et al., 2019).

De acuerdo con el informe anual elaborado por We Are Social \& Hootsuite (2020), se calcula que algo más de la mitad de la población a nivel mundial usa RRSS. Asimismo, se calcula que los usuarios tienden a usar estas redes alrededor de 2 horas y 24 minutos al día, siendo las más utilizadas Facebook, YouTube y WhatsApp (Espinel-Rubio et al., 2021).

En la actualidad, los adolescentes tienen la oportunidad de acceder a las RRSS con facilidad, favoreciendo su comunicación. De hecho, en el ámbito de las aplicaciones, cada día aparecen nuevas plataformas que fomentan el cambio en los hábitos de consumo (Establés et al., 2019). De acuerdo con el estudio llevado a cabo por Rodado et al., (2021), los jóvenes les conceden a las RRSS una gran importancia, ya que les permiten estar en contacto con otras personas que se encuentran lejos, así como para estar informados de lo que ocurre a su alrededor.

Según boyd \& Ellison (2007), las RRSS son multiplataformas que posibilitan la creación de perfiles online, donde los consumidores son los responsables de la configuración de su privacidad y delimitación de contactos. Las RRSS tienen una enorme trascendencia entre la juventud. Según Oberst et al. (2016), los jóvenes se exponen en este medio de una manera íntegra, asumiendo las formas de relación como un proceso natural dentro de su entorno de socialización, evolución y desarrollo (boyd, 2014).

\section{Influencia en adolescentes: actitud frente a los nuevos formatos}

Gracias a la digitalización y a la convergencia mediática se han iniciado nuevas formas de hacer y ver televisión, ofreciendo consumos individualizados y multiplataforma. Lo que se conoce como visionado conectado (connected viewing) genera unos hábitos a nivel mundial de entretenimiento multimedia, gracias a la unión de la tecnología digital y las RRSS, por un lado, y de los medios de comunicación tradicionales, por otro (Holt \& Sanson, 2014). 
Como consecuencia de los últimos cambios tecnológicos y empresariales, la televisión se ha visto en la necesidad de modificar su estructura. Elementos como la web $3.0 \mathrm{o}$ la hiperconectividad han contribuido a la modificación en la forma de consumir de los espectadores, resumiéndose en el principio de en cualquier lugar, en cualquier momento y en cualquier dispositivo (anywhere, anytime, any device), que caracteriza a la televisión líquida (Quintas-Froufe \& González-Neira, 2016). Debido a esta transformación, se han modificado elementos como el tiempo, el lugar y los emisores (Vázquez-Herrero et al., 2019). De acuerdo a un estudio reciente realizado, se ha podido constatar cómo las plataformas audiovisuales van ganando terreno en el consumo de los más jóvenes, pero la televisión sigue teniendo un papel fundamental entre ellos ocupando el primer lugar a la hora de consumir contenido audiovisual (Marcos-Ramos et al., 2020).

En este nuevo contexto existe una preocupación creciente por el uso que los adolescentes realizan de esta realidad actual. En diferentes investigaciones se ha demostrado cómo estos tienden a ver cada vez menos la televisión tradicional en beneficio de las nuevas plataformas, fomentando usuarios activos en relación a los productos audiovisuales. Según Vázquez-Herrero et al., (2019), los jóvenes son un sector de la población que ha crecido en un entorno digital, donde la interactividad es un factor esencial en su día a día, convirtiéndose en creadores de contenidos y reclamando algo más que un programa de televisión. Necesitan experimentar a nivel transmedia, es decir, unificar los distintos ámbitos en los que se fundamenta su vida y promover su competencia creadora de contenidos. Es fundamental impulsar otros instrumentos que confronten la realidad televisiva transmedia, del mismo modo que se requiere conocer cuáles son las habilidades de esta audiencia juvenil (Herrero-de-la-Fuente, 2017).

En relación a investigaciones realizadas en este campo, la oferta de las diferentes plataformas televisivas brinda la posibilidad de visionar contenidos bajo demanda (Becker et al., 2019). Otros analistas, como Núñez-Ladeveze et al. (2018), apuntan que desde el año 2012 se constata una disminución de la audiencia entre la población infantil y juvenil debido, entre otras causas, por el incremento del uso de teléfonos móviles y tablets.

Es evidente que la televisión ha sido modificada por las eventualidades entre espectadores, sobre todo, entre los más jóvenes. Habitualmente, la fragmentación del público se ha llevado a cabo por edades (Ferguson \& Greer, 2016), aunque en la actualidad, estos criterios están condicionados por el uso de las tecnologías (Hardey, 2011). Los adolescentes exploran nuevas vivencias a nivel comunicativo, ofreciéndoles la posibilidad de colaborar activamente en los diálogos, así como en el contenido (Gabardo, 2014).

\section{Objetivos}

El presente trabajo tiene la finalidad de conocer cuáles son los hábitos de consumo entre los jóvenes ante los nuevos formatos narrativos y su función como prosumidores frente a las RRSS. Para ello, se ha seleccionado la serie de ficción "Élite" como parte de la muestra de estudio. Estrenada en octubre de 2018, esta producción española ha sido producida por Zeta Producciones, y distribuida por la plataforma de vídeo bajo demanda Netflix. Cuenta con cuatro temporadas con ocho capítulos cada una. Actualmente, su emisión se ha extendido hasta 2022. 


\section{Diseño y método}

En relación al método, se ha optado por una metodología mixta, aplicando técnicas cualitativas y cuantitativas. Se considera metodología cualitativa "aquella que produce datos descriptivos: las propias palabras de las personas, habladas o escritas, y la conducta observable" (Taylor \& Bogdan, 1986, p. 20). Y, de acuerdo con Hueso \& Cascant (2012), la metodología cuantitativa emplea diferentes estrategias de carácter estadístico con el fin de analizar aspectos relevantes para el grupo objeto de estudio.

\subsection{Muestra e investigación cualitativa}

Por lo que respecta a los instrumentos de investigación y, en relación al método cualitativo, se ha llevado a cabo un análisis de contenido desde el punto de vista interpretativo. Casetti \& De-Chio (1991) definen el estudio de análisis como la comprensión e interpretación del texto con el fin de obtener el sentido de lo que se expone mediante una restauración personal, aludiendo a la experiencia y relativo al espectador. También López-Gutiérrez \& Nicolás-Gavilán (2016) hacen referencia al análisis interpretativo de las series, donde se tiene en cuenta tanto la intertextualidad, que se asienta sobre los referentes que posee el espectador, así como la ética de la serie, mostrando los valores, comportamientos y estilos de vida que se muestran en la misma.

Para realizar dicho análisis, el estudio se ha basado en las temáticas de la serie, tanto a nivel de intertextualidad como desde una perspectiva ética. La muestra se ha extraído a través del visionado de la totalidad de los 32 capítulos de la misma, con un total de 26 horas y 20 minutos, estableciendo una relación con las temáticas más recurrentes en cada temporada por capítulos. La información técnica de la serie puede ser consultada en el siguiente enlace: https://bit.ly/3yxcp2E

\subsection{Muestra e investigación cuantitativa}

A nivel cuantitativo se ha desarrollado una encuesta, definida por Groves et al. (2004, p. 4) como aquel "método sistemático para la recopilación de información de una muestra de los entes, con el fin de construir descriptores cuantitativos de los atributos de la población general de la cual los entes son miembros". El instrumento, conformado por un total de 17 ítems, se encuentra dividido en tres dimensiones diferenciadas: 1) Perfil socio-demográfico; 2) Hábitos de consumo en los medios audiovisuales; y 3) la serie "Élite": temáticas y redes. Dentro de los dos primeros bloques, los ítems se caracterizaron por ser preguntas cerradas, mientras que el tercer apartado está basado en la escala de Likert tipo 4 (donde 1 significa "totalmente en desacuerdo", 2 "en desacuerdo", 3 “de acuerdo" y 4 “totalmente de acuerdo"). La encuesta utilizada para esta investigación se puede encontrar en el siguiente enlace: https://bit.ly/3eUkkOD

Con el objetivo de contrastar la utilidad de la encuesta y su adecuación al tema de estudio al que se refiere, se ha convenido la realización de un control íntegro por parte de seis expertos en la materia, aportando todos ellos información útil y necesaria para la conformación de la misma. Con la ayuda del programa estadístico SPSS 27, se creó una matriz con el propósito de calcular la fiabilidad de acuerdo con el Alpha de Cronbach, siendo el resultado final ,875. A través de la prueba piloto realizada por los expertos, este dato corroboró una consistencia interna altamente fiable, introduciendo un instrumento adecuado para el análisis de los objetivos previamente delimitados.

La muestra total de la encuesta asciende a 505 adolescentes con edades comprendidas entre los 12 y los 18 años. En la presente investigación han participado dos centros educativos, de manera voluntaria, estando uno de ellos situado en la provincia de Al- 
mería. En ambos casos, tanto los participantes en este estudio como sus padres/madres o tutores legales fueron informados del objetivo y desarrollo de la investigación. Este análisis está basado en un estudio de aproximación al fenómeno, debido a que se trata de una muestra determinada y limitada en el número de participantes; aun así, es iniciática, permitiendo asentar las bases para futuras investigaciones.

\section{Resultados}

\subsection{Análisis cualitativo}

En relación al análisis cualitativo se han visionado los 32 capítulos de las cuatro temporadas de la serie con el fin de conocer cuáles son sus temáticas. Al tratarse de una serie dirigida, sobre todo, a un público adolescente, las historias están directamente relacionadas con el mundo juvenil.

En relación a la presencia y frecuencia de temáticas, cabe señalar que, en las tres temporadas, se constata cómo los siguientes temas tienen una presencia en todos los capítulos: 1) Diferencias sociales y/o culturales entre los jóvenes de distintas clases sociales; 2) Deseo de integración; 3) Enfrentamiento y rivalidad entre clases sociales diferentes; 4) Deseo de superación tanto académica como social; 5) Amor y amistad entre clases sociales distintas; 6) Exigencias familiares hacia los adolescentes y relaciones homosexuales. Además, se observa que, a pesar de no existir los siguientes tópicos en todos los capítulos, tienen un peso específico y relevante a lo largo de toda la serie: a) Celebración de fiestas, donde el alcohol y las drogas tienen una gran presencia; b) Es recurrente apelar al trabajo entre los adolescentes de clases media-baja, provocando una clara diferenciación entre los jóvenes; c) El uso de las RRSS entre los adolescentes es un hecho factible en la serie como medio de comunicación entre ellos; d) Se presentan diferentes tipos de familias (monoparentales, tradicionales u homoparentales); e) El hecho de presentar diferentes clases sociales provoca que se generen situaciones de discriminación entre ellas; f) Las tradiciones sociales y/o culturales tienen un gran peso específico como la religión y tratar de seguir las creencias que esta impone; g) Asimismo, que la trama gire en torno a un asesinato provoca que se traten temas como la cárcel, el chantaje, la extorsión e, incluso, la mentira. 
Imagen 1. Nube de palabras con los temas principales de la serie Élite

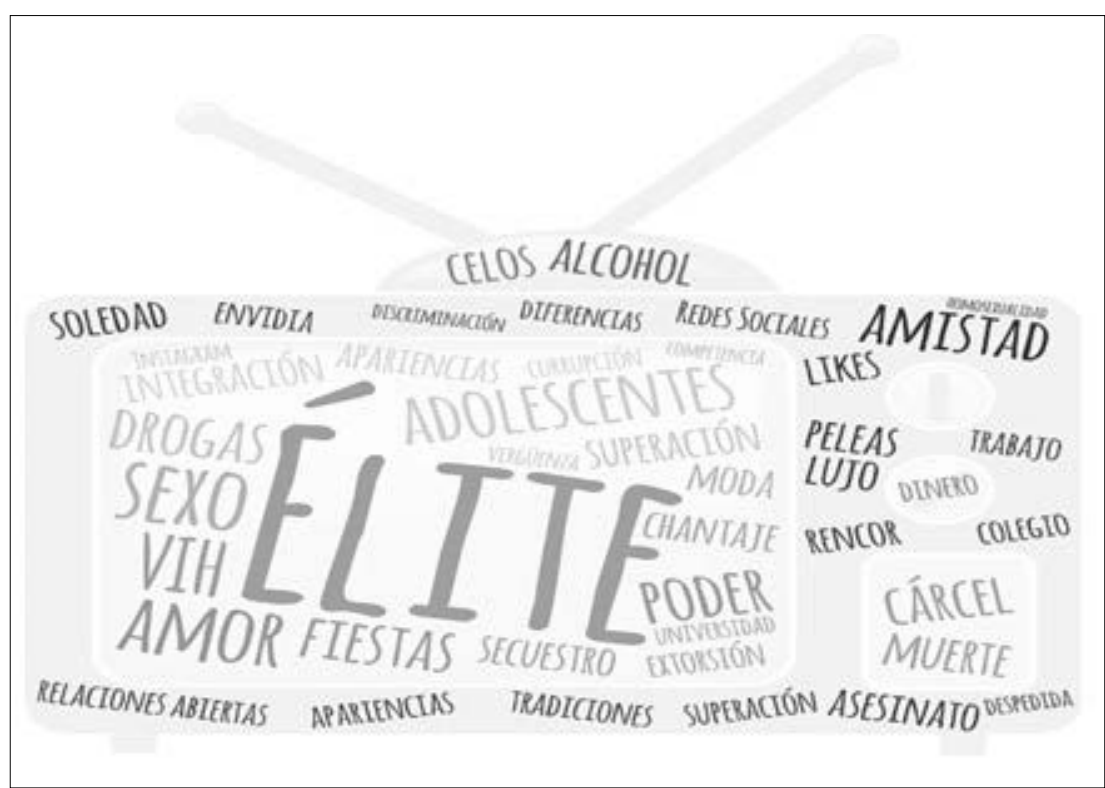

Fuente. Elaboración propia

\subsection{Análisis cuantitativo}

Para llevar a cabo la investigación se arrojó datos esenciales sobre los hábitos de consumo que los jóvenes presentan ante el consumo de los medios audiovisuales. En último término en este estudio, se trató de indagar acerca de las temáticas de la serie "Élite" y su relación con las RRSS.

\subsubsection{Perfil socio-demográfico y hábitos de consumo}

En la primera dimensión se ha realizado un análisis socio-demográfico, así como un estudio de los hábitos de consumo de los medios audiovisuales entre los adolescentes con el fin de conocer el perfil de los encuestados. Por ello, se han planteado diferentes cuestiones relacionadas con el género, la edad, el tipo de centro donde se estudia, los estudios que realizan y las actividades que desarrollan en su tiempo libre, así como contenidos audiovisuales que consumen, los formatos escogidos para ello y el número de horas y series que ven en total.

En lo que al género se refiere, 267 participantes son mujeres (52,9\%). El rango de edad más numeroso es el de 16 años con un total de 121 encuestados (24\%) y un total de 323 de participantes (64\%) viven en la Comunidad Autónoma de Andalucía, convirtiéndose en la mayor representación en este estudio. En lo referente a lo académico, 364 del total $(72,1 \%)$ acude a centros concertados y un total de 307 (60,8\%) cursa estudios de Educación Secundaria Obligatoria. Con respecto a las actividades que prefieren realizar 
en su tiempo libre, 113 de los jóvenes (21\%) se inclinan por ver series de televisión, seguido por la opción de escuchar música con un total de 98 adolescentes $(19,4 \%)$

En relación a los hábitos de consumo de los medios audiovisuales entre los adolescentes, 248 (49,1\%) de los encuestados se decantan por ver series de televisión y $268(53,1 \%)$ visionan los contenidos a través de las plataformas de pago. Atendiendo al número de horas de consumo, se puede observar una diferencia entre semana frente al fin de semana, ya que 254 (50,3\%) de la población participante admite ver una media de dos horas al día la televisión durante la semana, subiendo el consumo hasta cuatro horas o más con un total de 339 (67,1\%) durante el fin de semana (Gráfico 1).

Gráfico 1. Horas de visionado televisión

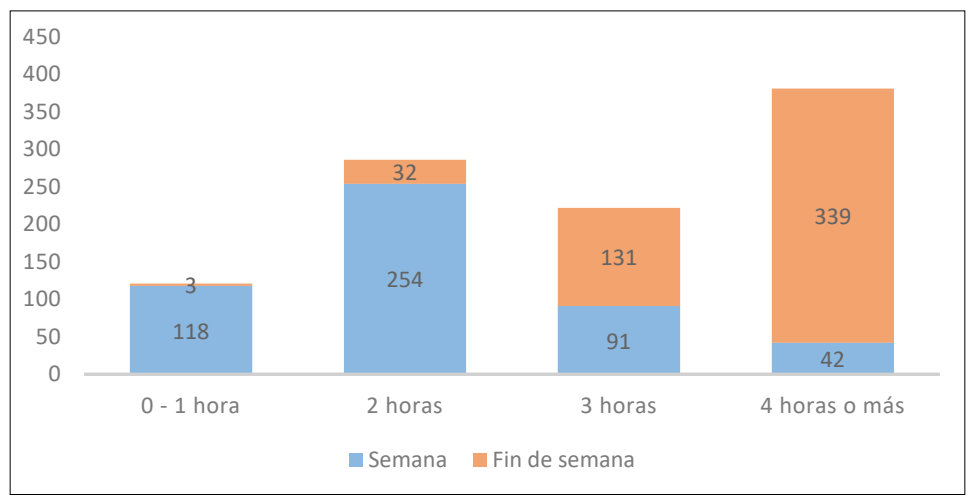

Fuente. Elaboración propia

Por último, sobre las series de televisión que actualmente visionan, cabe destacar que un total de 465 (92,1\%) admite que consumen series a diario, y 233 del total $(46,1 \%)$ sigue cuatro o más series de televisión, seguido por 108 (21,4\%) que señalan ver un total de dos series.

\subsubsection{Temáticas de la serie Élite y las RRSS}

En esta tercera y última dimensión se ha procedido a dar respuesta a los interrogantes sobre la serie Élite, su temática y las RRSS. Para ello, se ha utilizado la herramienta estadística SPSS con el fin de calcular tanto la media como la desviación típica a las preguntas realizadas y efectuar un análisis descriptivo de los resultados. Se debe mencionar que 366 (72,5\%) del total de los encuestados admite que conoce la serie y, por lo tanto, serán estos los datos analizados.

Concerniente a la pregunta sobre si la serie "Élite" refleja la realidad diaria de los adolescentes en la actualidad. Tan solo 70 $(19,1 \%)$ de ellos reconoce que sí lo hace, y 116 (31,6\%) admiten que tal vez muestre la realidad de los jóvenes.

En relación a los temas que se ofrecen en la serie y que suscitan el interés entre los encuestados, se presentan valores correspondientes a la media en torno a 2,7. Por su parte, en relación a la desviación típica, los valores son cercanos a uno, por lo que no existen diferencias estadísticas significativas. En correspondencia a los valores más elevados cabe destacar los ítems "Asesinatos/ Robo/Cárcel” (3,34), seguido de “Corrupción/Chantaje/Extorsión” (3,12) y “Amor" (3,03). Por otro lado, los valores descendentes 
son "Disfunción familiar" (2,18), seguido de "La religión y los jóvenes" (2,30) y, por último, "Enfermedades sexuales/VIH/Sida" $(2,46)$. En síntesis, se puede concluir que los adolescentes tienen interés por los temas que se tratan dentro de la serie, destacando la temática relacionada con los asesinatos, el robo y/o la cárcel (Tabla 1).

Tabla 1. Temáticas relacionadas con la serie Élite

\begin{tabular}{|c|c|c|c|c|c|}
\hline & $\mathrm{N}$ & Mínimo & Máximo & Media & Desv. típ. \\
\hline Mentiras/Rivalidad & 366 & 1 & 5 & 2,91 & ,879 \\
\hline Redes sociales/Identidad digital & 366 & 1 & 5 & 2,95 & ,867 \\
\hline Poder económico & 366 & 1 & 6 & 2,98 & 1,002 \\
\hline Asesinatos/Robos/Cárcel & 366 & 1 & 6 & 3,34 & ,888 \\
\hline Inclusión/Exclusión social & 366 & 1 & 6 & 2,53 & ,926 \\
\hline Superación social/académica & 366 & 1 & 6 & 2,59 & ,916 \\
\hline Alcohol/Drogas/Fiesta & 366 & 1 & 5 & 2,97 & 1,049 \\
\hline Enfermedades sexuales/VIH/Sida & 366 & 1 & 5 & 2,46 & 1,061 \\
\hline Amor & 366 & 1 & 5 & 3,03 & ,968 \\
\hline Relaciones homosexuales/LGTB & 366 & 1 & 5 & 2,52 & ,970 \\
\hline La religión y los jóvenes & 366 & 1 & 5 & 2,30 & ,882 \\
\hline Sexo/ Relaciones abiertas/Embarazos adolescentes & 366 & 1 & 5 & 2,88 & 1,074 \\
\hline Corrupción/Chantaje/Extorsión & 366 & 1 & 5 & 3,12 & ,942 \\
\hline Presión social & 366 & 1 & 5 & 2,66 & ,914 \\
\hline Disfunción familiar & 366 & 1 & 4 & 2,18 & ,811 \\
\hline $\mathrm{N}$ válido (según lista) & 366 & & & & \\
\hline
\end{tabular}

Fuente. Elaboración propia a partir de los datos obtenidos del SPSS 
Para continuar y, según se plantea en la siguiente cuestión, las RRSS o herramientas que utilizan los adolescentes para comunicarse, hay que destacar que la media presenta valores dispares. Los ítems que presentan una mejor valoración son WhatsApp $(3,85)$ y YouTube $(3,44)$. Por el contrario, Snapchat $(2,10)$ y Twitter $(2,15)$ son los menos valorados. Por otra parte, en la desviación típica se puede observar una alteración de los resultados en torno a la media; mientras que la mayoría de los ítems están en torno a 1, apreciando una desviación en relación a WhatsApp $(, 531)$ y YouTube $(, 718)$. De este análisis, se deduce que la herramienta preferida como medio de comunicación entre los adolescentes es WhatsApp (Tabla 2).

Tabla 2. RRSS o herramientas para la comunicación

\begin{tabular}{|l|r|r|r|r|r|}
\hline & $\mathrm{N}$ & Mínimo & Máximo & Media & Desv. típ. \\
\hline Facebook & 366 & 1 & 5 & 2,62 & 1,173 \\
\hline Instagram & 366 & 1 & 6 & 3,42 &, 920 \\
\hline Twitter & 366 & 1 & 5 & 2,15 &, 955 \\
\hline Snapchat & 366 & 1 & 5 & 2,10 &, 957 \\
\hline Youtube & 366 & 1 & 5 & 3,44 &, 718 \\
\hline Whatsapp & 366 & 1 & 6 & & 3,85 \\
\hline N válido (según lista) & 366 & & & & \\
\hline
\end{tabular}

Fuente. Elaboración propia a partir de los datos obtenidos del SPSS

Con respecto al tercer y último aspecto, se analizó la relación de la serie con las RRSS. En lo que a la desviación típica se refiere, los valores son cercanos a uno, por lo que cabe mencionar que no se encuentran diferencias estadísticas significativas. Al atender a la media, se pueden observar valores dispares. Los ítems que presentan una mejor puntuación son "Considero importante que la serie de televisión esté representada a través de las redes sociales" $(3,24)$, seguido por "Me intereso a través de las Redes Sociales por indagar más sobre la serie (historia, personajes...)" $(3,10)$ y "A través de estas cuentas he comenzado a seguir otros perfiles relacionados con la serie/temática" $(2,97)$. Por otro lado, aquellos peor puntuados son "He aumentado mi visibilización (número de seguidores) por participar en las redes sociales de la serie" $(1,81)$, "He realizado conexiones con otros usuarios/fans de la serie" $(2,01)$ y, por último, “Comparto publicaciones y produzco nuevos contenidos relacionados con la serie” (2,10). En suma, los adolescentes estiman que es de gran relevancia que la serie de televisión tenga presencia en las RRSS (Tabla 3). 
Tabla 3. Élite y las RRSS

\begin{tabular}{|c|c|c|c|c|c|}
\hline & $\mathrm{N}$ & Mínimo & Máximo & Media & Desv. típ. \\
\hline $\begin{array}{l}\text { Considero importante que la serie de televisión esté representada a } \\
\text { través de las redes sociales }\end{array}$ & 366 & 1 & 6 & 3,24 & ,876 \\
\hline Sigo las cuentas (Facebook, Instagram y Twitter) de la serie & 366 & 1 & 5 & 2,65 & ,969 \\
\hline Leo post y veo vídeos a través de las redes sociales de la serie & 366 & 1 & 5 & 2,90 & ,889 \\
\hline $\begin{array}{l}\text { Interactúo a través de "likes" en cada una de las cuentas activas de } \\
\text { la serie }\end{array}$ & 366 & 1 & 6 & 2,59 & ,977 \\
\hline $\begin{array}{l}\text { Comparto publicaciones y produzco nuevos contenidos relaciona- } \\
\text { dos con la serie }\end{array}$ & 366 & 1 & 4 & 2,10 & ,843 \\
\hline He realizado conexiones con otros usuarios/fans de la serie & 366 & 1 & 5 & 2,01 & ,828 \\
\hline $\begin{array}{l}\text { He aumentado mi visibilización (número de seguidores) por partici- } \\
\text { par en las redes sociales de la serie }\end{array}$ & 366 & 1 & 5 & 1,81 & ,756 \\
\hline $\mathrm{N}$ válido (según lista) & 366 & & & & \\
\hline
\end{tabular}

Fuente. Elaboración propia a partir de los datos obtenidos del SPSS

En definitiva, es necesario resaltar que el perfil de los participantes en esta investigación responde al de una mujer de 16 años que reside en la Comunidad Autónoma de Andalucía y cursa estudios de Educación Secundaria Obligatoria en un centro público. La actividad preferida entre la población encuestada es la de ver series de televisión, y los programas más consumidos son, en este orden, las series ficcionales. Visionan una media de cuatro o más series y utilizan las plataformas de pago. El consumo medio de contenidos audiovisuales entre semana es de dos horas, alzándose a cuatro o más durante el fin de semana. Asimismo, la herramienta preferida para comunicarse es WhatsApp. En relación a la serie "Élite", no se corresponde con la realidad de los adolescentes en la vida diaria, siendo las temáticas más relevantes de la misma los asesinatos, el robo y la cárcel. En relación a las RRSS, se considera fundamental que la serie tenga presencia en las mismas. 


\section{Discusión y conclusiones}

Según un estudio, en torno a las series para adolescentes producidas durante los años 90 y principios del siglo XXI, se constató una sucesión de características concretas determinadas por ser producciones procedentes de países anglófonos, dramáticas y con una duración que oscilaba entre 40 y 60 minutos. El principal propósito era reproducir la vida diaria de sus jóvenes personajes bajo las temáticas de las relaciones personales, del amor y de la amistad (Fedele et al., 2011). De acuerdo con Fedele (2014), las series actuales se caracterizan por el género dramático y de ciencia ficción, así como un ambiente recurrente que gira en torno a centros educativos y hogares de los protagonistas. Así queda reflejado en la serie Élite, donde hemos podido constatar que son frecuentes los escenarios dentro del centro escolar, así como en las casas de los personajes. Asimismo, y siguiendo el autor, las series españolas tienden a buscar argumentos que reflejen la realidad de los adolescentes como, por ejemplo, buscar y conocer su propia identidad o iniciar las primeras relaciones amorosas. Por otra parte, según Carrera et al., (2020), hoy en día se tiende a consumir contenido audiovisual siguiendo los códigos establecidos por el fenómeno fandom, estableciendo conexiones tanto online como offline por parte de los seguidores de la serie, fomentando una comercialización aún mayor del producto. Así queda reflejado en el presente estudio, ya que tal y como nos han hecho saber los encuestados, utilizan las RRSS para conocer más sobre la serie y los actores, o entrar en contacto con otros usuarios que también siguen estos medios.

Con respecto al objetivo propuesto sobre cuáles son los hábitos de ocio y tiempo libre de los jóvenes, los adolescentes tienden a aprovechar su tiempo de ocio haciendo actividades diversas, destacando el visionado de series de televisión, mientras que la segunda opción más escogida es escuchar música. Dentro de los contenidos audiovisuales elegidos, destacamos que cerca de la mitad de la población encuestada prefiere ver series de televisión, siendo las plataformas de pago el medio seleccionado por la mayoría de ellos para visionar este contenido. Actualmente, la sociedad evoluciona gracias a que está conectada a su entorno más inmediato y la realidad de los adolescentes se genera a través de los medios de comunicación. Internet consigue generar distintos ambientes propicios para el aprendizaje mediante diferentes prácticas innovadoras y discursos digitales.

En cuanto al número de horas que los jóvenes destinan a ver la televisión, existe una clara diferencia entre el tiempo que dedican durante la semana frente al fin de semana. En este sentido, durante la semana consumen una media de dos horas al día, mientras que el fin de semana aumenta a cuatro horas. La gran mayoría de la población encuestada admite ver series de televisión a diario y algo menos de la mitad confiesa seguir cuatro o más series actualmente. La evolución del tratamiento de las audiencias mediáticas le ha concedido un rol activo al público en lo que a la apropiación y elaboración de mensajes audiovisuales se refiere (Sullivan, 2013; Zeller et al., 2014). La clave para poder comprender este desarrollo es la colaboración y la función de las audiencias adolescentes, tanto en la recepción como en la elaboración de mensajes que se generan en los medios de comunicación. Esto conlleva a que los adolescentes convivan en su día a día con los medios y por ello, sean capaces de interactuar con los mismos (de-la-Fuente-Prieto et al., 2019). Por este motivo, son fundamentales aquellas investigaciones acerca de una correcta alfabetización entre los jóvenes (Lacasa-Díaz, 2018; Livingstone \& Sefton, 2016).

En relación a la serie objeto de estudio en esta investigación, "Élite", una gran mayoría admite conocerla: sin embargo, llama la atención cómo tan solo un porcentaje pequeño el que considera que refleja la realidad de los adolescentes de hoy en día. Con respecto a las temáticas que trata la serie, el aspecto que más interesa a los adolescentes es aquel relacionado con asesinatos, robos y/o cárcel. 
Al hablar de las RRSS, WhatsApp es elegida por los participantes como el medio de comunicación por excelencia, siendo Instagram la red social preferida por estos. Al cuestionar la relación existente entre las RRSS y la serie de televisión, los adolescentes reflejan que es muy importante que esta tenga presencia en las mismas. Por ello, lo mostrado en esta investigación refleja la relevancia que tienen en la actualidad para los adolescentes los medios audiovisuales, donde las plataformas de pago tienen un peso específico dentro de ellos, priorizando los contenidos relacionados con el entretenimiento como las series de televisión. Asimismo, se ha podido constatar como las RRSS juegan un papel primordial en la vida diaria de los más jóvenes. Cabe destacar los datos extraídos del estudio realizado por IAB Spain (2020) en relación al uso de las RRSS, se llegó a la conclusión que la sociedad usa con frecuencia las RRSS, siendo WhatsApp, Instagram y Facebook las plataformas más demandadas por su carácter espontáneo y con información actualizada.

\section{Limitaciones y líneas de investigación}

En relación a las limitaciones, nos propusimos realizar un estudio entre adolescentes, es decir, entre 12 y 18 años, puesto que los personajes de la serie se encuentran en esa franja de edad. Sin embargo, en ciertos momentos, nos encontramos ante la situación de no obtener el número representativo deseado.

En relación a la prospectiva, se plantean una serie de cuestiones, las cuales podrían ser objeto de estudio con el fin de completar y extender la presente investigación, tales como: conocer los roles que se presentan en las series de televisión dirigidas para adolescentes, saber si los personajes presentados están estereotipados en relación a cuestiones de género, edad, condición social..., averiguar si adoptan los adolescentes estos modelos y los extrapolan a su vida diaria, conocer qué opinión generan las series que se emiten sobre los jóvenes entre la sociedad, o investigar si reflejan realmente la vida de un adolescente ajeno a la ficción. Estas proposiciones facilitan nuevas vías de estudio que determinen la relación dicotómica entre juventud y producción serializada a nivel nacional.

\section{Agradecimientos}

Este artículo ha sido traducido al inglés por Maite Bell y revisado/editado por Laura Dixon.

\section{Referencias bibliográficas}

Anstead, N., \& O'Loughlien, B. (2011). The emerging viewertariat and BBC question time: Television debate and real time commenting online. The International Journal of Press/Politics, 16(4), 440-462. https://doi.org/10.1177.1940161211415519

Awidi, I.T., Paynter, M., \& Vujosevic. T. (2019). Facebook group in the learning design of a higher education course: An analysis of factors influencing positive learning experience for students. Computers \& Education, 129, 106-121. https://doi.org(10.1016/j. compedu.2018.10.018

Bardin, L. (1996). Análisis de contenido. Akal. 
Becker, V., Abreu, J., Nogueira, J., \& Cardoso, B. (2018). O desenvolvimento da TV não linear e a desprogramação da grelha. Observatorio (OBS) Journal, 12(1), 199-216. https://doi.org/10.15847/onsOBS12120181007

Berelson, B. (1952). Content analysis in communication researches. Glencoe III, Free Press.

boyd, D. (2014). It's complicated. Y.U. Press.

boyd, D., \& Ellison, N.B. (2007). Social network sites: Definition, history and scholarship. Journal of computer - mediated communication, 13(1), 210-230. https://doi.org/10.1111/j.1083-6101.2007.00393.x

Brenley, D.B., \& Covey, J. (2018). Risky behavior via social media: The role of reasoned and social reactive pathways. Computers in huuman behaviour, 78, 183-191. https://doi.org/10.1016/j.chb.2017.09.036

Burgess, J. \& Green, J. (2009). YouTube: Online video and participatory culture. Polite Press.

Capapé, E. (2020). Nuevas formas de consumo de los contenidos televisivos en España: una revisión histórica (2006 - 2019). Estudios sobre el mensaje periodístico, 26(2), 451-459. https://doi.org/10.5209/esmp6773

Carrera, P., Blanco-Ruiz, M. \& Sainz-de-Baranda-Andújar, C. (2020). Consumo mediático entre adolescentes. Nuevos medios y viejos relatos en el entorno transmedia. Historia y comunicación social, 25(2), 563-574. https://doi.org/10.5209/hics.72285

Casetti, F., \& Di Chio, F. (1991). Cómo analizar un film. Paidós Ibérica.

Crusafon, C.; González-Saavedra, C., \& Murciano, M. (2020). Televisión pública en España y jóvenes universitarios: radiografía del acceso, consumo y valores más preciados. En “Comunicación y diversidad. Selección de comunicaciones del VII Congreso Internacional de la Asociación Española de Investigación de la Comunicación (AE-IC). Valencia, 28 - 30 de octubre, 357 - 366. https://doi.org/10.3145/AE-IC-epi.2020.e21

De-La-Fuente-Prieto, J., Lacasa-Díaz, P., \& Martínez-Borda, R. (2019). Adolescentes, redes sociales y universos transmedia: la alfabetización mediática en contextos participativos. Revista Latina de Comunicación Social, 74, 172-196. https://doi.org/10.4185/ RLCS2019-1326

Díaz-Gandasegui, V. (2011). Mitos y realidades de las redes sociales. Prisma Social, 6, 340-366. http://bit.ly/39WY06T

Diego-González, P., Guerrero-Pérez, E., \& Etayo-Pérez, C. (2014). Televisión conectada en España: contenidos, pantallas y hábitos de visionado. Revista Mediterránea de Comunicación, 5(1), 179-199. https://doi.org/10.14198/MEDCOM2014.5.1.10

Espinel-Rubio, G.A., Hernández-Suárez, C.A., Prada-Núñez, R. (2021). Usos y gratificaciones de las redes sociales en adolescentes de educación secundaria y media: de lo entretenido a lo educativo. Encuentros, 19(1), 137-156. https://doi.org/10.15665/ encuen.v19.i01.2552

Establés, M.J., Guerrero-Pico, M., \& Contreras-Espinosa, R.S. (2019). Jugadores, escritores e influencers en redes sociales: procesos de profesionalización entre adolescentes. Revista Latina de Comunicación Social, 74, 214-236. https://doi.org/10.4185/ RLCS-2019-1328

Fedele, M. (2011). El consum adolescent de la ficció serieada televisiva. (Tesis Doctoral). Universitat Autónoma de Barcelona. Barcelona, España. http://bit.ly/2wJPhUl 
Fedele, M. (2014). Los personajes jóvenes en la ficción televisiva: identidades, modelos y representaciones juveniles en la era digital. Memorial Final de Proyecto Alianza 4 Universidades. Departamento de Comunicación, Universitat Pompeu Fabra.

Fedele, M., Prado, E., \& García-Muñoz, N. (2011). La singularidad de las series juveniles producidas a partir de los años noventa. En J. Sierra-Sánchez \& S. Liberal-Ormaechea (Coords.). Investigaciones educomunicativas en la sociedad multipantalla. (pp. 325344). Fragua.

Ferguson, D.A., \& Greer, C.F. (2016). Reaching a moving target: how local TV statios are using digital tools to connect with generation C. International Journal on Media Management, 18(3-4), 141-161. https://doi.org/10.1080/14241277.2016.1245191

Flores-Cueto, J., Morán-Corzo, J., \& Rodríguez-Vila, J.J. (2009). Las redes sociales. Boletín electrónico de la Unidad de Virtualización Académica. Universidad de San Martín de Porres.

Galán, E., \& del-Pino, C. (2010). Jóvenes, ficción televisiva y nuevas tecnologías. Área Abierta, 25, 1 - 17. http://bit.ly/2wJPhUl

Gallego, F. (2013). Social TV analytics: nuevas métricas para una nueva forma de ver televisión. Index.comunicación, 3(1), 13 - 39. http://bit.ly/3j8W39z

Garabardo, J.A. (2014). Los internautas españoles: quienes son y quienes no son. Revista Anuncios, 1489, 41. https://bit.ly/39YfIHb Gil-Quintana, J., \& Fernández-Galiano-Amorós, M. (2020). Publicaciones, interacciones, verdades y mentiras de adolescentes españoles en Instagram. Belo Horizonte, 13(1), 20 - 44. https://doi.org/10.17851/1983-3652.13.1.20-44

Groves, R.M., Fowlere, F.J., Couper, M.P., Lepkowski, J.M., Singer, E., \& Tourangeau, R. (2004). Survey methodology. John Wiley \& Sons.

Hardey, M. (2011). Generation C: Content, creation, connections and choice. International Journal of Markey Research, 53(6), 749-770. https://doi.org/10.2501/IJMR-53-6-748-770

Hernández, K., Yanez, J., \& Carrera, A. (2017). Las redes sociales y adolescencias. Repercusión en la actividad física. Universidad y Sociedad, 9(2), 242-247. https://bit.ly/3gTYqOr

Herrero-De-La-Fuente, M. (2017). Nuevas fórmulas para la televisión en directo: el uso de Facebook Live en Atresmedia. Miguel Hernández Communication Journal, 8, 521 - 563. https://doi.org/10.21134/mhcj.v0i8.202

Holt, J., \& Sanson, K. (2014). Connected viewing: Selling, streaming \& sharing media in the digital era. Routledge

Hueso, A., \& Cascant, M.J. (2012). Metodología y técnicas cuantitativas. De investigación. Universitat Pollitècnica de València

IAB (2020). Estudio Anual Redes Sociales 2020. Elogia. http://bit.ly/3rdbYIt

Jenkins, H. (2006). Convergence culture: Where old and new media collide. New York University Press.

Jódar-Marín, J.Á. (2019). Los nuevos formatos audiovisuales en los cibermedios: del reportaje televisivo al videonews. Communication \& Society, 32(4), 63-75. https://bit.ly/2D6VBZz

Lacasa-Díaz, P. (2018). Expresiones del futuro: cómo se comunicarán las nuevas generaciones. Morata.

Lamelo-Varela, C. (2016). Televisión social y transmedia. Editorial UOC.

Livingstone, S.M., \& Sefton-Green, J. (2016). The class: Living and learning in the digital age. NYU Press. 
López-Gutiérrez, M.L. \& Nicolás-Gavilán, M.T. (2016). El análisis de series de televisión: construcción de un modelo interdisciplinario. ComHumanitas: Revista Científica de Comunicación, 6(1), 22-39. http://bit.ly/3nEiNR5

Marcos-Ramos, M., Pérez-Alaejos, M.P.M., Cerezo-Prieto, M., \& Hernández-Prieto, M. (2020). Infancia y contenidos audiovisuales online en España: una aproximación al consumo y a la mediación parental en las plataformas OTT. Icono 14, $18(2), 245$ - 268. https://doi.org/10.7195/ri14.v18i2.1560

Masanet, M.J., \& Fedele, M. (2019). El “chico malote” y la “chica responsable": modelos aspiracionales y representaciones juveniles en las teen series españolas. Palabra Clave, 22(2), 1-27. https://doi.org/10.5294/pacla.2019.22.2.5

Medina, M., Herrero, M. \& Portilla, I. (2019). La evolución del mercado de la televisión de pago y del perfil de los suscriptores. Revista Latina de Comunicación Social, 74, 1751 - 1780. https://doi.org/10.4185/RLCS-2019-1409

Morimono, S.A., \& Friedland, L.A. (2011). The lifeworld of youth in the information society. Youth and Society, $43(2), 549$ - 567. https://doi.org/10.1177/0044118X10383655

Núñez-Ladeveze, L., Núñez-Cana, M., \& Irisarri-Núñez, J.A. (2018). Pautas de integración cultural y política de la sociedad de medios masivos en la sociedad red. Revista Latina de Comunicación Social, 73, 184 - 207. https://doi.org/10.4185/RLCS-2018-1252 Oberst, U.; Chamarro, A., \& Renau, V. (2016). Gender stereotypes 2.0 - Self - representations of adolescents on Facebook. Comunicar, 48(24), 81-89. https://doi.org/10.3916/C48-2016-08

Oliva-Marañón, C. (2012). Redes sociales y jóvenes: Una intimidad cuestionada. Aposta: revista de ciencias sociales, 54,1 - 16. https://bit.ly/3p1W5Ci

Pavón-Arrrizabalaga, A., Zuberogoitia, A., Astigarraga, I., \& Juaristi, P (2016). Consumo de series de televisión de los adolescentes en la era de la digitalización audiovisual: prácticas y motivaciones. Dígitos. Revista de Comunicación Digital, 2, 35 - 51. http://bit. ly/2wJPhUl

Pereira-López, M., Mo-Groba, D. \& Lagares-Díez, N. (2019). Información off-line y on-line en campaña: uso de las redes sociales en las elecciones catalanas 2017. RISTI, 26, 229-241. https://bit.ly/3wX7Mzt

Prado, E. (2013). La televisió: un univers en mutació. L’Espill, 43, 129-137. La televisió: un univers en mutació.

Quintas-Froufe, N., \& González-Neira, A. (2016). Consumo televisivo y su medición en España: camino hacia las audiencias híbridas. El profesional de la información, 25(3), 376-383. https://doi.org/10.3145/epi.2016.may.07

Rodado, Z., Jurado, L., \& Giraldo, S. (2021). Uso excesivo de las redes sociales en adolescentes de 10 a 14 años, un riesgo latente de la nueva era. Revista Estudios Psicológicos, 1(1), 33-49. https://doi.org/10.35622/j.rep.2021.01003

Ruano-López, S., Fernández-Falero, M.R. \& Garcés-Botacio, I. (2016). Las series de televisión en las redes sociales: estudio de caso. Opción, 12, 525 - 540. https://bit.ly/2OawWbL

Saavedra-Llamas, M., Papí-Gálvez, N., \& Perlado-Lamo, M. (2020). Televisión y Redes Sociales: las audiencias sociales en la estrategia publicitaria. El profesional de la información, 29(2), 1-14. https://doi.org/10.3145/epi.2020.mar.06

Sánchez-Noriega, J.L. (1997). Crítica de la seducción mediática: comunicación y cultura de masas en la opulencia informativa. Tecnos. 
Selva, D. (2016). Social television: Audience and political engagement. Television and new media, 17(2), 159-173. https://doi. org/10.1177/1527476415616192

Shapiro, L.A.S., \& Margolin, G. (2014). Growing up wired: Social networking sites and adolescent psychosocial development. Clinical child and family psychology review, 17(1). https://doi.org/1.1007/s10567-013-0135-1

Sullivan, J.L. (2013). Media audiences: Effects, users, institutions, and power. SAGE.

Taylor, S. \& Bogdan, R.C. (1989). Introducción a los métodos cualitativos de investigación. Paidós.

Tse, Y. (2016). Television's changing role in social togetherness in the personalized online consumption of foreign TV. New Media \& Society, 18(8), 1547 - 1562. https://doi.org/10.1177/1461444814564818

Vázquez-Herrero, A., González-Neira, N. \& Quintas-Froufe, N. (2019). La audiencia activa en la ficción transmedia: plataformas, interactividad y medición. Revista Latina de Comunicación Social, 74, 73-93. https://doi.org/10.4185(RLCS-2019-1322

Vila, P.; Barceló, T. \& Fuentes, G. (2015). La televisión inteligente en el nuevo espacio televisivo. El espectador activo y los nuevos lenguajes. En M. Francés; G. Llorca \& À. Peris, La televisión conectada en el entorno transmedia. (pp. 27-41). EUNSA, Ediciones de la Universidad de Navarra.

We Are Social \& Hootsuite (2020). Digital 2020. Global digital overview. Essential insights into how people around the world use the Internet, mobile devices. social media, and ecommerce. https://bit.ly/3DBhNpj

Zeller, F.E., Pontee, C.E., \& O’Neill, B.E. (2014). Revitalising audience research: Innovations in European audience research. Routledge.

Zorrilla-Abascal, M.L. (2012). Las competencias mediáticas para el siglo XXI según Henry Jenkins. Universidad Autónoma del Estado de Morelos. 